\title{
Picosecond timescale Raman processes and spectroscopy *
}

\author{
C. K. Johnson, G. A. Dalickas, S. A. Payne and R. M. Hochstrasser \\ Department of Chemistry, University of Pennsylvania, Philadelphia, PA 19104
}

\begin{abstract}
Time resolved properties of vibrational states of molecules on the picosecond scale are surveyed using both conventional spontaneous Raman effects as well as coherent Raman processes. In certain respects the coherent signal methods have been found to have distinct advantages related to their intrinsic time gating capability but they can have disadvantages in the form of nonresonant background interference. New picosecond time resolved Raman spectra of excited states and of myoglobins are presented.
\end{abstract}

\section{INTRODUCTION}

In chemistry and biology there are many intrinsically fast processes whose study requires subnanosecond spectroscopic methods. These processes include excited state dynamics as well as the evolution and decay of reaction intermediates. Optical spectroscopy is presently a routine method (1) for studying picosecond processes but vibrational spectroscopy presents more difficulties for the experimentalist. In this talk I will survey some recent efforts in our laboratory to obtain time resolved properties of vibrational states of molecules.

There were previous attempts to obtain vibrational spectra of molecules in solution with picosecond time resolution. One approach has involved comparing the Raman spectra obtained from focused with those from unfocused picosecond laser beams (2). This technique allows the detection of transient Raman spectra but does not provide much information about their time dependence. Quite recently a pump-probe Raman instrument was developed on the basis of a synchronously pumped and amplified dye laser driven by a cw modelocked argon laser (3). It is fair to say that the state of the art in picosecond time resolved Raman spectroscopy represents an early stage in the development of this method. Difficulties still exist in regard to wavelength tunability, time resolution, and detection. On the otherhand, for infrared spectroscopy there has been as yet no report of a subnanosecond time resolved method. So the situation with Raman methodology is much further advanced.

In principle, both coherent and incoherent Raman methods may be employed to detect short lived species. In certain respects the coherent signal methods have distinct advantages related to their intrinsic time gating capability, but they can have disadvantages in the form of nonresonant background interference.

In this talk I will describe our recent work using both conventional spontaneous Raman effects and coherent Raman processes to expose dynamical processes related to molecular vibrational states on the picosecond timescale.

\section{TIME RESOLVED SPONTANEOUS RAMAN SCATTERING}

The importance of time-resolved spontaneous Raman spectroscopy in better understanding structural dynamics was first stressed in a series of papers by Delhaye and co-workers (4-7). These investigators recognized the potential of three-dimensional imaging devices in obtaining very weak light scattering spectra and had moderate success in recording Raman spectra of molecules in solution using a Ruby laser as the source and a Vidicon as the detector. The laser and Vidicon technology have improved markedly since these early experiments.

*

* This research was supported by a grant from the Division of Research Resources of NIH (RP-01348-02) and in part by an NIH grant GM12592 and an NSF Grant (CHE 83-03916). 


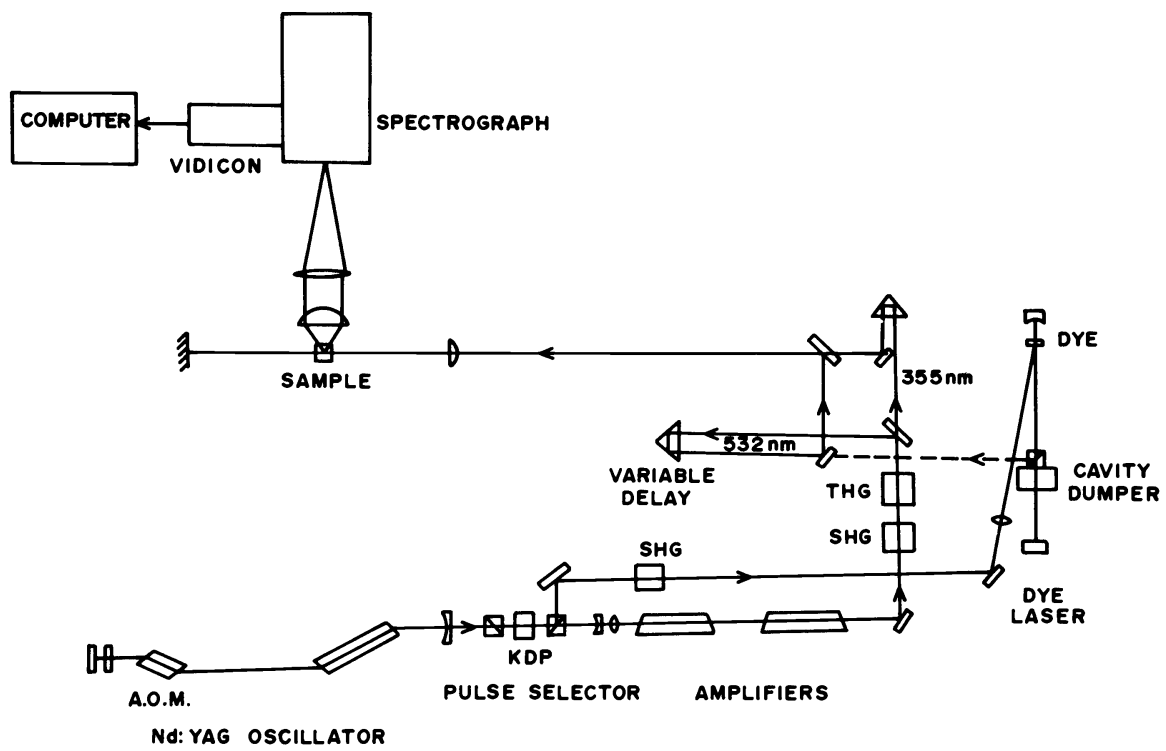

Figure 1. Picosecond time-resolved Raman spectrometer. The passively and actively (by an acousto-optic modulator) modelocked oscillator produces pulsetrains from which single pulses are selected, expanded, and amplified. The rejected pulsetrains can be used to pump a dye laser. Harmonics of the Nd:YAG laser are generated at $532 \mathrm{~nm}$ and $355 \mathrm{~nm}$. The $532 \mathrm{~nm}$ pulse is delayed with respect to the $355 \mathrm{~nm}$ and probes the sample region excited by the $355 \mathrm{~nm}$ pulse. Raman scattering is collected, dispersed, and imaged on a cooled SIT vidicon. Data is transferred to a microcomputer for processing.

Recent studies with nanosecond YAG-lasers, actively modelocked ND:YAG, and synchronously pumped dye lasers have strengthened the conviction that time-resolved Raman studies can make a significant impact on many areas of research (8). The development of nanosecond systems for Raman scattering is already quite advanced. Recently Terner, Spiro, Nagumo, Nicol and E1 Sayed (2) have carried out a time gated Raman experiment on HbCo photo products with spectra averaged over tens of picoseconds. In a similar experiment Hayward, Carleen, Siegman and Stryer (9) have used a high repetition rate Nd:YAG laser to observe the Raman spectrum of rhodopsin time gated at ca. $40 \mathrm{ps}$. In each of these experiments resolved transient vibrational spectra were observed as a result of optical pumping and the results used to claim incisive structural interpretations of the transients. It is apparent also that in each case the experiments did not have sufficient time resolution, flexibility in the variation of delay times, spectral extent and accuracy, or signal-to-noise to allow other than tentative interpretations of the results. However these important contributions and the earlier experiments pioneered by Delhaye and coworkers do signal the outstanding potential of time-resolved Raman spectroscopy. The importance of obtaining Raman spectrometers

\section{Figure 2}

Raman spectrum of benzophenone in ethanol. Top: ground-state benzophenone and solvent (EtOH) bands with $532 \mathrm{~nm}$ excitation. Bottom: Raman scattering from sample pumped at 355 nm. Pump-induced bands are resonance-enhanced.

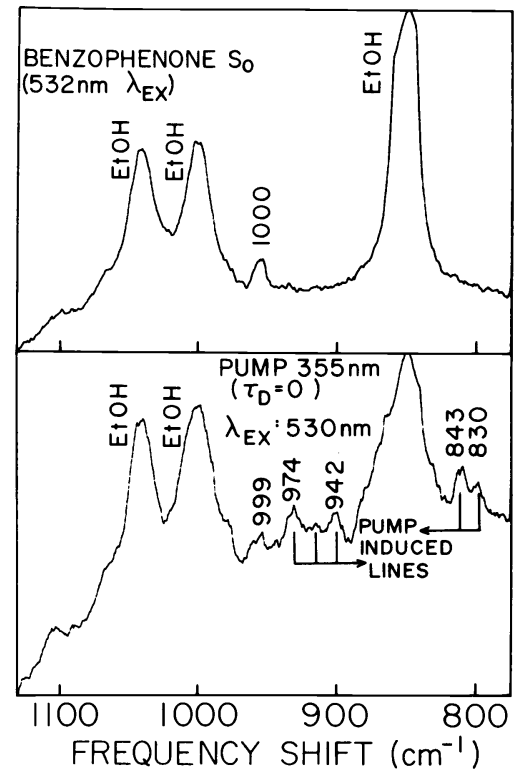


that do not have many of the disadvantages of these approaches is therefore evident.

In one approach we have used a moderately high repetition rate Nd:YAG 1aser. The laser consists of an oscillator amplifier design which provides a versatile source of both pump and probe radiation. The oscillators and amplifiers run at rep rates up to $20 \mathrm{~Hz}$. A pulse from theoscillator train is isolated by a Pockels cell before being amplified three times, as indicated schematically in Figure 1 .

The oscillator consists of an active-passive modelocked system which generates pulse trains with ca. $4 \mathrm{~mJ}$ in 20 pulses. The extracted pulse is amplified to ca. $10 \mathrm{~mJ}$. The experiments reported here use the $2^{\text {nd }}$ and $3^{\text {rd }}$ harmonics as the pump and probe pulses. The oscillator pulse beam quality is close to $\mathrm{TEM}_{\infty}$ as judged by far field distributions. The beam quality is vitally important in picosecond Raman experiments using intense pulses since it is only then that undesirable nonlinear optical effects can be controlled. Dynamical change can be probed simultaneously with the excitation pulse, or following it by up to several nanoseconds. Spontaneous Raman scattering generated in the sample is collected with $\mathrm{f} / 1$ optics and focused onto the slit of a polychromator. The dispersed spectrum, spanning region of about $400 \mathrm{~cm}^{-1}$, is imaged onto the face of a multichannel detector (vidicon intensified photodiode array). The detector is cooled to allow collection of signal for several minutes at a time with negligible dark current. Data from the vidicon is digitized and transferred to a laboratory computer for processing.

The system we have developed is unique in several respects. First, efficient laser pumping cavities have been used, allowing a high repetition rate at moderate flash-lamp powers. Second, the excitation and probe pulses are separate in time with a variable delay, in contrast to transient Raman systems which use the same pulse for both photoexcitation and probing. Our system allows spectra to be obtained at delays from the picosecond to the nanosecond time regimes, allowing a link to be established with nanosecond time resolution studies performed elsewhere. Third, our excitation pulse is intense enough to transfer large fractions of the sample molecules within the excitation volume to the excited state. This is a significant advantage over continuous-wave synchronously pumped laser system, where the excitation pulse energy is lower by several orders of magnitude. At the same time, our probe beam average power of ca. 10 milliwatts is comparable to that often used in steady-state Raman spectrometers.

The effectiveness of our spectrometer in obtaining Raman spectra in the pump-probe mode on the picosecond timescale is illustrated by the study of benzophenone. It is known that $353 \mathrm{~nm}$ excitation of benzophenone results in the formation of a ${ }^{1} \pi *$ state which lives for ca. 15 ps in benzene solution. The decay of this state corresponds to intersystem crossing to the ${ }^{3} \pi^{*}$ state (10) which displays a transient absorption in the visible. This transient absorption was previously studied by Weisman, Greene and Hochstrasser (1) using a picosecond generated continuum. The Raman spectrum shown in Figure 2 was obtained by pumping with $353 \mathrm{~nm}$ and probing with $530 \mathrm{~nm}$. We have thought that the new transitions, indicated on the Figure, correspond to triplet state vibrations since the spectrum is very similar at 0 and 100 ps delay. Although the singlet state is known to absorb $530 \mathrm{~nm}$ light $(1,11)$ the absorption coefficient of the triplet is apparently considerably larger, resulting in the resonance enhancement of its Raman spectrum but not that of the singlet. The observed vibrational transitions do not correspond to any of those seen in the singlet (ground state) $\rightarrow$ triplet absorption spectrum (12). This is consistent with our expectation that the resonant transition is $n^{*} \rightarrow \mathrm{n} \pi^{*}$ in which $\mathrm{C}=0$ stretching associated modes do not have large FranckCondon factors in contrast to the case of $\mathrm{A} \rightarrow \mathrm{n} \pi^{*}$ excitation. The indicated modes are therefore assigned as aromatic skeletal vibrations associated with the nearby modes.

Another example of the current capability of the Raman spectrometer concerns our studies, done in collaboration with T. G. Spiro and S. Dasgupta, of myoglobin derivitives. Figure 3 shows the picosecond laser excited spectra of $\mathrm{Mb}$ and $\mathrm{MbCO}$. Experiments were carried out with $\mathrm{MbCO}$ using on $1 \mathrm{y} 530 \mathrm{~nm}$ radiation and using both $353 \mathrm{~nm}$ pulses with delays. In this case the $530 \mathrm{~nm}$ pulse alone is able to partially bleach the MbCO due to the photodissociation of CO following light absorption in the visible (Q-band) spectral region of the heme. However with $530 \mathrm{~nm}$ alone, the MbCO bands indicated on the Figure, are still clearly evident. This is a result of incomplete photobleaching of the MbCO by the $0.4 \mathrm{~mJ} 530 \mathrm{pu} 1 \mathrm{se}$. This causes difficulties in the assignment of band positions due to $\mathrm{Mb}^{+}$, the initially created ligandfree structure. The absorption coefficient of $\mathrm{MbCO}$ at $353 \mathrm{~nm}$ is ca. 2.5 times that at $530 \mathrm{~nm}$. so that when the sample is first excited by a $353 \mathrm{~nm}$ pulse of $\mathrm{mJ}$ energy, the bleaching is more complete than with a $530 \mathrm{~nm}$ pulse of about the same energy. For this reason the spectrum on Figure 3 (c) corresponding to $353 \mathrm{~nm}$ excitation and $530 \mathrm{~nm}$ probing with 30 ps delay shows significantly less MbCO scattering and thus better resolution of the $\mathrm{Mb}^{+}$core marker bands.

An interesting aspect of the new results presented in the foregoing paragraph concerns the change in vibrational frequencies of $\mathrm{Mb}^{+}$compared with those of stable $\mathrm{Mb}$, as indicated in Figure 3. We have shown earlier that the isobestic point in the picosecond time resolved absorption spectrum of $\mathrm{Mb} / \mathrm{MbCO}$ obtained $6 \mathrm{ps}$ after $353 \mathrm{~nm}$ excitation, is shifted by 
Figure 3

Resonance Raman spectrum of myoglobin. Top: Deoxymyoglobin with $532 \mathrm{~nm}$ excitation. Middle: Carboxymyoglobin with $532 \mathrm{~nm}$ excitation. Bottom: Carboxymyoglobin pumped at $355 \mathrm{~nm}$, probed at $532 \mathrm{~nm}$ at 30 ps time delay.

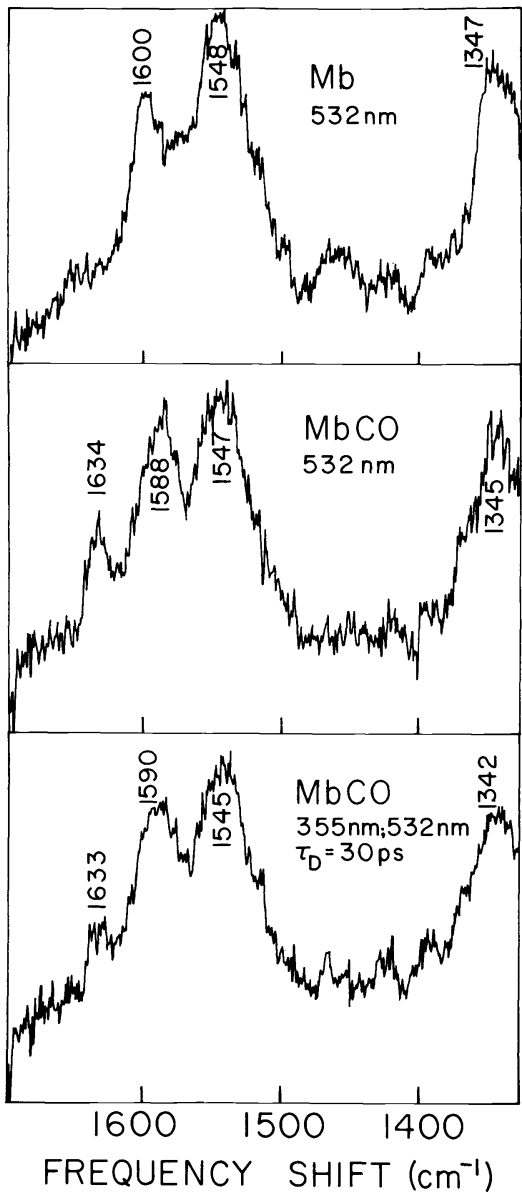

$+1.5 \mathrm{~nm}$ from its location in the equilibrium mixture (13). On the otherhand recent nanosecond transient absorption studies have shown that at ca. $10 \mathrm{~ns}$ the optically induced difference spectrum is identical with the stable one (14). In addition the nanosecond time resolved Raman spectra (15) show that the generated Mb has a vibrational spectrum which is the same as that for stable $\mathrm{Mb}$. These results indicate that a small change in heme structure takes place on the timescale ca. $10 \mathrm{ps}$ to ca. $10 \mathrm{~ns}$. This evolution extends to longer times than would allow excited electronic states to be invoked.

\section{Coherent Raman Methods with Picosecond Time Resolution (16)}

In coherent methods such as CARS and RIKES (17) the signal pulse is generated with a well defined direction and frequency. These techniques are useful probes of metastable species or excitations. In a typical experiment a system would be pumped to generate the transients and at a later time probed by the appropriate set of pulses to generate the coherent signal resulting from the transient. The time resolution in this case is determined by the convolution of pump and probing pulses.

Goldberg (18) has reported the use of a picosecond pulse generated continuum and a $\mathrm{Nd}$ :Glass fundamental as a CARS probe. The $527 \mathrm{~nm}$ pulse corresponds to the $\omega_{1}$ field and the continuum to $\omega_{2}$, and resonances were seen at frequencies $2 \omega_{1}-\omega_{2}$ where $\omega_{1}-\omega_{2}$ corresponded to a vibrational frequency. Our approach has been to use a synchronously pumped dye laser instead of the continuum.

The CARS apparatus shown in Figure 4 is based on a Nd:glass laser system which operates at $0.5 \mathrm{~Hz}$ repetition rate. The dye laser is driven by anplified pulsetrain and a single pulse is cavity dumped from its synchrony with the extraction of a pulse from the pulsetrain. The extracted $1054 \mathrm{~nm}$ pulse is amplified and used to generate laser harmonics for the experiments. A $527 \mathrm{~nm}$ pulse $\left(\omega_{1}\right)$ reaches the sample time coincident with the broadband dye laser pulse $\left(\omega_{2}\right)$ and $s$ spectrograph Reticon combination is used to observe the spectrum generated in the region $2 \omega_{1}-\omega_{2}$. The FWHM of the CARS peak is instrument limited by the resolution of the spectrograph and the $\omega_{1}$ beam. 


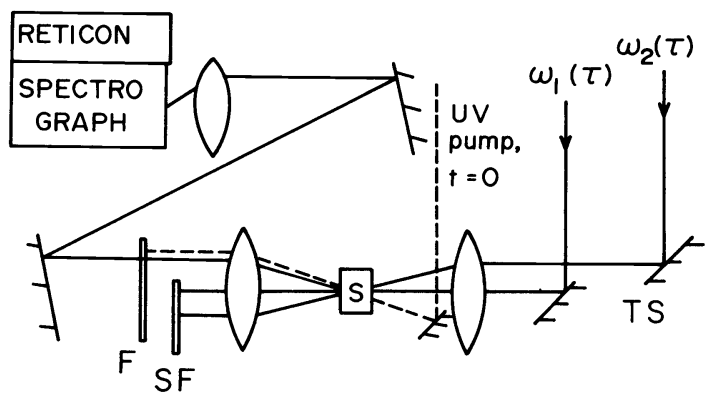

Figure 4. Picosecond multiplexed CARS set-up. A broadband dye laser output serves as the $\omega_{2}$ beam while a doubled amplified laser pulse is the $\omega_{1}$ beam. A dye laser beam is 2 reflected from mirror on translation stage (TS) to adjust phase-matching angle. UV (fourth harmonic) focused separately to avoid chromatic aberration, (UV not used for data in Fig. 5). $\omega_{1}$ and $\omega_{2}$ are timed to be coincident and delayed relative to the UV pump. Signal is spatially and spectrally filtered (SF and F) before entering Spex Doublemate polychromater and is then detected by PAR 1420 reticon in conjunction with the 1218 Controller and the 1215 Console.
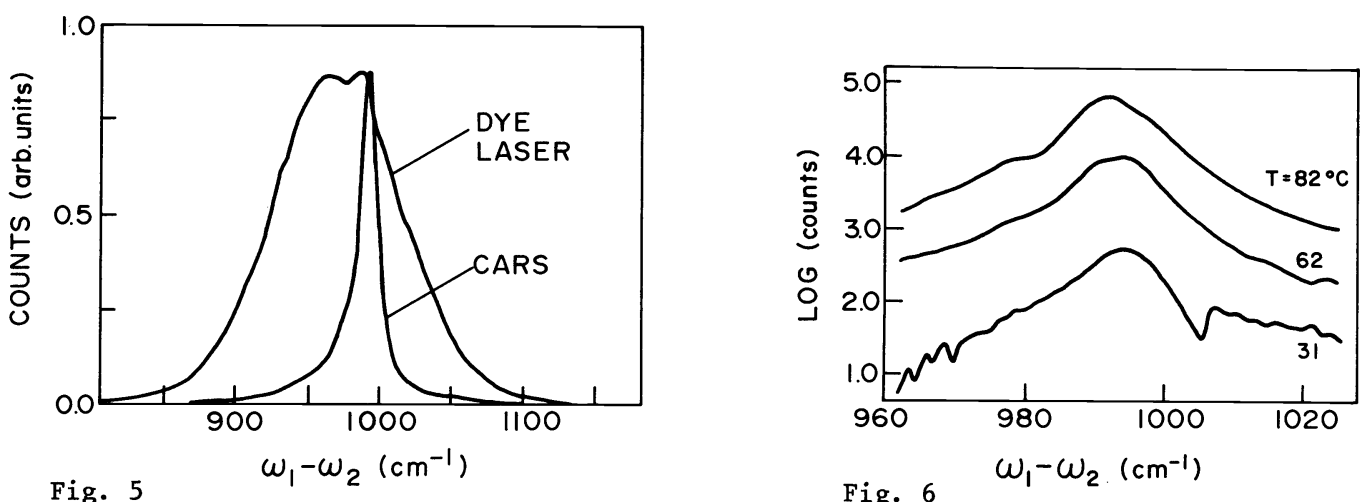

Figure 5. Picosecond multiplexed CARS spectrum of $992 \mathrm{~cm}^{-1}$ mode of liquid benzene resulting from 50 laser shots in $0.2 \mathrm{~nm}$ pathlength cell, superimposed with the R6G dye laser spectral output.

Figure 6. Picosecond multiplexed CARS spectra of gaseous benzene resulting from 15 laser shots and the use of an $8 \mathrm{~cm}$ cell. Spectra are not normalized to the dye laser output. The cell was held at $100^{\circ} \mathrm{C}$ while the benzene reservior temperature was varied as indicated in the figure. The curves display the expected squared dependence on benzene vapor pressure.

Figure 5 shows a spectrum of liquid benzene taken in the region of the $9.92 \mathrm{~cm}^{-1}$ mode using this arrangement. Figure 6 shows the CARS spectrum of gaseous benzene at three pressures, taken with a colinear geometry of the probing beams.

This type of CARS spectroscopy is different from the arrangement in which the coherence is probed. A three pulse coherence experiment in which the sample is first prepared in metastable (usually vibrationa1) states by means of two pulses $\omega_{1}$ and $\omega_{2}$, and then probed by a pulse at $\omega_{1}$ delayed appropriately, measures the decay of the coherence of the transient. The coherence decay constant $1 / \mathrm{T}_{2}$ is composed of a sum of the population decay, $1 / 2 \mathrm{~T}_{1}$, and the pure dephasing, $1 / \mathrm{T}_{2}$. At ambient temperatures and for vibrational transients, we usually have that $\mathrm{T}_{2}{ }^{2}<\mathrm{T}_{1}^{2}$ so that this technique measures essentially the pure dephasing. On the otherhand at sufficiently low temperatures $\mathrm{T}_{2}{ }^{\prime} \rightarrow \infty$ and the coherence decay measures the decay of the population of transients.

Techniques which probe the coherence are not useful for the study of population dynamics in liquids and solution simply because $\mathrm{T}_{2}{ }^{\prime}<<\mathrm{T}_{1}$ in such cases. However in homogeneous media at low temperatures this inequality is reversed and coherence probing could become the method of choice for the study of vibrational spectra. In particular, we have studied the vibrational states of molecular crystals at low temperatures using time resolved CARS methods (19). In this experiment the coherence persisting in vibrational transitions as a result of their preparation is probed by a delayed picosecond pulse. The presence of coherence is evidenced by a signal on the antistokes side of the probe pulse. The vibrational spectrum in this instance is observed in Fourier transform by a quantum beat pattern in the antiStokes signal versus probe delay (20). 
This technique should become extremely useful for time resolved vibrational spectroscopy using femtosecond laser pulses (21) for preparing and probing. The excitation process will then be faster than coherence loss even at more elevated temperatures, and the effective spectral range of the Fourier transform Raman spectrum will be a few thousand $\mathrm{cm}^{-1}$. A great advantage of the coherent methods is that they allow the transient Raman spectrum to be recorded even in the presence of omnidirectional spontaneous fluorescence.

Acknowledgements: The work on MbCO was carried out in collaboration with the Princeton group and will be reported later in a joint publication by C. K. Johnson, G. A. Dalickas, S. Dasgupta, T. G. Spiro and R. M. Hochstrasser.

\section{$\underline{\text { References }}$}

(1) R. B. Weisman, B. I. Greene and R. M. Hochstrasser, J. Chem. Phys. 70,1247 (1979).

(2) J. Terner, T. G. Spiro, M. Nagumo, M. F. Nicol and M.A. E1 Sayed, J. Amer, Chem. Soc., $101,3238(1980)$.

(3) T.L. Gustafson, D. M. Roberts and D. A. Chernoff, J. Chem. Phys. 79, 1559 (1983).

(4) M. Delhaye, Applied Optics Z, 2195 (1968).

(5) M. Delhaye and P. Dhomelincourt, J. Raman Spect. 3 , 33 (1975).

(6) M. Bridoux and M. Delhaye, Nouv. Rev. d'Optique App1. 1, 23 (1970).

(7) M. Delhaye, "Time and Spatially Resolved Raman Spectroscopy" in Lasers in Physical Chemistry and Biophysics (ed. J. Joussot-Dubien, E1sevier 1975) p213.

(8) "Time Resolved Vibrational Spectroscopy" ed. G. H. Atkinson (John Wiley \& Son, New York 1983).

(9) G. Hayward, W. Carlsen, A. Siegman and L. Stryer, "Picosecond Resonance Raman Spectroscopy: The Initial Photolytic Event in Rhodopsin and Isorhodopsin" in Picosecond Phenomena II (Eds. Hochstrasser, Kaiser and Shank, Springer Series in Chemical Physics 14, 1980; p. 377).

(10) R. W. Anderson, R. M. Hochstrasser and G. W. Scott, Chem. Phys. Letts. 28,153 (1974).

(11) K. J. Choi, L. A. Halliday and M. R. Topp in "Picosecond Phenomena II" (Eds. R. M. Hochstrasser, K. Kaiser and C. V. Shank, Springer Verlag 1980) p. 131.

(12) R. M. Hochstrasser, M. Schafer and S. Dym, J. Chem. Phys. 48,646 (1968).

(13) P. A. Cornelius and R. M. Hochstrasser, in "Picosecond Phenomena III" (Eds. K. B. Eisentha1, R. M. Hochstrasser, W. Kaiser and A. Laubereau; Springer-Ver1ag 1982) p. 288 .

(14) E. R. Henry, J. H. Sommer, J. Hofrichter and W. A. Eaton, J. Mo1. Biol. 166, $443(1983)$.

(15) J. M. Friedman, R. A. Stepnosky, M. Stavola, M. R. Ondrias and R. L. Cohn, Biochem. $\underline{21}, 2022$ (1982); T. G. Spiro and S. Dasgupta, private communication.

(16) S. Payne and R. M. Hochstrasser, work in progress.

(17) See e. g. "Nonlinear Spectroscopy" by M. D. Levinson (Academic Press, New York, 1982).

(18) L. S. Goldberg, in Picosecond Phenomena III (eds. K. B. Eisenthal, R. M. Hochstrasser, W. Kaiser and L. Laubereau, Springer-Verlag, 1982) p. 94.

(19) F. Ho, W.-S. Tsay, J. Trout and R. M. Hochstrasser, Chem. Phys. Letts. 83, 5 (1981).

(20) S. Velsko, J. Trout and R. M. Hochstrasser, J. Chem. Phys. 79, 2114 (1983).

(21) R. L. Fork, B. I. Greene and C. V. Shank, App1. Phys. Letts. 38, 671 (1981); C. V. Shank, R. L. Fork and R. T. Yen in "Picosecond Phenomena III (Eds. K. B. Eisenthal, R. M. Hochstrasser, W. Kaiser, A. Laubereau; Springer-Verlag 1982) p. 2. 